\title{
A STUDY OF STUDENTS' SENSE OF LEARNING COMMUNITY IN ONLINE ENVIRONMENTS
}

\author{
Peter Shea
}

University at Albany - State University of New York

\begin{abstract}
This paper looks first at some of the often unspoken epistemological, philosophical, and theoretical assumptions that are foundational to student-centered, interactive online pedagogical models. It is argued that these foundational assumptions point to the importance of learning community in the effectiveness of online learning environments. Next, a recent study of 2314 online students across thirty-two college campuses is presented. This study reports on learners' sense of community and it is concluded through factor and regression analysis that elements of the Community of Inquiry model [1]—specifically learners' recognition of effective “directed facilitation" and effective instructional design and organization on the part of their instructor contributes to their sense of shared purpose, trust, connectedness, and learning - core elements of a community of learners. Gender also appears to play a small role in students' sense of learning community with female students reporting higher levels than their male classmates. Implications for online learning environments design are discussed.
\end{abstract}

\section{KEYWORDS}

Learning Community, Sense of Community, Community of Inquiry

\section{INTRODUCTION}

To make rational choices about any pedagogical strategy or approach in teaching and learning, it is helpful to examine the underlying epistemological, philosophical, and theoretical assumptions that such strategies and approaches reflect. This process applies equally to online and traditional instruction, but is particularly useful in new learning environments in which there remains a sense of uncertainty about "what works" and why. This process is useful because it helps us to understand the likely outcome of educational practices in new environments and allows us to communicate these expectations before the practices are "inflicted" upon unsuspecting students.

There has been an increasing interest in the development of online communities of learners in recent years, especially in higher education - a development that reflects some fairly specific epistemological, philosophical, and theoretical assumptions. There is a growing consensus [2, 3, 4, 5] that we can, in online environments, create online learning communities - "groups of people, connected via technology-mediated communication, who actively engage one another in collaborative, learner-centered activities to intentionally foster the creation of knowledge, while sharing a number of values and practices" [6]. To understand online learning communities as a goal for higher-education online learning environments, it is useful to examine the foundations and assumptions upon which online learning communities rest. These include three related changes - a philosophical shift from objectivism towards constructivism; a theoretical shift from behaviorism towards socio-cognitive views of education; and a pedagogical shift from direct instruction to the facilitation of collaborative learning. Each of these is outlined in more detail below. 
The philosophical and epistemological shift referred to here is the change in views of reality as existing "out there" separate from the mind of the individual learner and towards a conception of reality as constructed by the learner through interaction with the world and others in it. This paradigm shift is reflected in the utilization of learning communities in traditional and online learning environments as follows. If individuals construct reality through interaction with the world and others, then it makes sense to design environments where that construction is more easily accomplished. If learners do not "receive" knowledge through direct encoding of objective reality or through its transmission from a more knowing instructor, but rather co-create understanding with the assistance of instructors, (and parents and more able peers and so on), then it makes sense to facilitate this co-construction of knowledge though the design of the learning environment. The philosophical assumption reflected in the pedagogical approach that seeks to foster online communities of learners is this-that reality is not "out there" waiting to be poured into learners, but rather that each of us constructs a version of reality that is situated in a context of culturally-mediated activity - of interaction in specific kinds of setting and institutions. Online learning communities, it is assumed, can be designed to represent good and supportive examples of such settings, and such community-centered approaches represent a vast, and philosophically-corresponding, improvement to instructor or lecture-centered models.

The notion of a supportive learning community reflects what we have learned about "the nature of individual minds operating in an enabling culture" [7] in the decades that have passed since the "cognitive revolution" - the paradigmatic shift in how we think about (among other things) learning. The change is one of emphasis-now commonly placed on understanding the mind and its underlying psychological processes rather than on observable changes in behavior without regard for the "black box" of the mind. The educational emphasis on communities of learners reflects the underlying assumptions about knowledge and learning that have come out of this transition in psychology away from behaviorism and towards socio-cognitive views in a number of ways. The recognition that we, as a species, live in communities and understand our world through mental states developed in joint activity with others is a critical facet of the shift. There is, by now, a longstanding, underlying assumption that the tenets of behaviorism - with their emphasis on overt activities and the shaping of behavior towards desired endsare insufficient to facilitate or understand learning in all its complexity and nuance. The design of online, collaborative-learning environments is founded on the assumption that culture matters, that we live and learn in community for a reason, that community-based living and meaning-making is a reflection, to a large degree, of our unique genetic makeup as a species. As Bruner [7] puts it,

...not only do we represent the world in our own minds (replete with meaning), but we respond with preternatural sensitivity to the way that world is represented in the minds of others. And by virtue of that sensitivity we form a representation of the world as much from what we learn about it through others as from responding to events in the world directly. Our worlds then, are vicarious to a degree unthinkable in any other species.”

Concern with this "preternatural sensitivity" to the representations in the mind of others-this speciesdefining vicariousness is a foundation of socio-cognitive views of knowledge making, quite distinct from behavioral antecedents - and also foundational to learning community-based models of online education.

The third shift which is assumed in community based, online teaching and learning models is the change in emphasis from direct instruction to facilitated learning. This transition in pedagogical approach, which has been ongoing in traditional classroom-based learning environments for decades [8, 9], is itself an outcome of the epistemological and philosophic changes noted above. The transition from teaching to learning as a primary goal of education assumes that students construct and hold greater responsibility for their own learning and that the traditional, lecture-based model—based on a discredited behavioral paradigm, fails to effectively consider and support the pedagogical processes involved in knowledge building. 
We are concerned with the development of shared goals, trust, and mutual support-features of high functioning communities - because those characteristics lay the foundation for an effective pedagogy of constructivism-one which values, encourages and sustains productive discourse. It is through the thoughtful design of opportunities to actively participate in such discourse that learners can engage in the pedagogical processes that support learning. These processes include scaffolded opportunities to: articulate and present current views, consider alternative views expressed by classmates and instructors, reflect and re-think, and integrate new ideas into existing cognitive structures. The distinction in pedagogical models that encourage and utilize learning community is in the conception of learningwhich may be considered a process of reflection in action: action extends thought-reflection is shaped by the consequences of action [10]. At their best, online learning-community models allow participants to actively engage one another in ideas and perspectives they hold to be educationally worthwhile, exciting, and provocative. It is through the design of the learning environment, with an emphasis on shared educational goals, support, collaboration, and trust that these processes can be most effectively and functionally activated.

There is a great deal of work to be done to understand whether and how online-learning community models work. Coming to a common understanding of what a learning community is has been a longstanding challenge. Measuring an abstract concept such as community is not trivial. In the remainder of this paper I will describe some previous [11, 12] and more recent attempts [13] to define and measure students' sense of community, satisfaction, and learning in a large asynchronous learning network, which has, through faculty development efforts, worked to convey to instructors the importance of community to effective online education.

In addition to the challenge of coming to a common understanding of what defines an online learning community is choosing an appropriate mechanism by which to measure learner's sense of community according to such a definition. Another critical issue is to understand the factors that contribute to a sense of community in online environments. If we believe that online learning-community models reflect appropriate philosophies, theories and pedagogies, and if we accept that it is through well designed methods that the social and cognitive processes of learning are facilitated in online environments, then understanding learners' sense of community in online environments relative to these methods is essential. It should be noted here that we need to recognize that this is a critical piece of a larger puzzle-that what we are trying to understand in this research is how an environment for constructivist learning can be designed and orchestrated, but that we are not directly measuring learning itself-but rather online participants recognition of connectedness, cohesion, and shared learning goals, and an assessment of their instructors' behaviors that lead to these.

To define and evaluate online learners' feelings and recognition of learning community we need an instrument designed to measure these constructs. Rovai $[14,15]$ developed and refined an instrument to assess college students' sense of learning and community, the Classroom Community Scale, in which he defines a learning community along several dimensions-cohesion, spirit, trust, interaction, interdependence and shared educational goals. The Classroom Community Scale contains twenty items and measures learning community through two subscales-connectedness and learning. The connectedness subscale reflects respondents' feelings regarding cohesion, spirit, trust, and interdependence. The learning subscale reflects the degree to which respondents shared educational goals and benefits through their interaction with other course participants. We believe that the instrument is a good measure of the characteristics of a learning community-i.e. students' sense of trust, belongingness, and mutual support in the pursuit of shared educational goals. So, to assess student's sense of learning and community we used Rovai's Classroom Community Scale [14, 15], which we will refer to as a measure of "learning community" - the dependent measure for our recent work. 
The Community of Inquiry Model provides the framework from which we developed the independent variables for the study. In this model Garrison and colleagues [1] present an interlocking set of factors that cohere in the creation of a community of learners. These include various forms of "presence" that need to be established in online environments, among them teaching presence, social presence, and cognitive presence. In the research conducted here we focused specifically on how teaching presence [16] — defined as effective instructional design and organization, the facilitation of discourse, and direct instruction-influences online students' sense of learning community. We developed a survey instrument to measure students' perceptions of teaching presence as the independent variable in this research. The survey contained seventeen items to assess instructional design and organization, facilitation of discourse, and direct instruction. The scale assessing instructional design and organization has six items which reflect the setting of curriculum, the design of methods, establishment of time parameters, effective utilization of the medium, and the establishment of "netiquette". The "facilitation-of-discourse" section contained six items assessing the professor's proficiency in identifying areas of agreement and disagreement; seeking to reach consensus and understanding; encouraging, acknowledging, and reinforcing student contributions; setting the climate for learning; drawing in participants and prompting discussion; and assessing the efficacy of the instructional process. The direct-instruction section has five items assessing the professor's proficiency in presenting content and questions, focusing the discussion on specific issues, confirming understanding, diagnosing misperceptions, and injecting knowledge from diverse sources. The rating of the teaching presence components was on a five-point Likert type scale from strongly disagree $=0$, disagree $=1$, neutral $=2$, agree $=3$, to strongly agree $=4$.

Additional items on the survey asked about students' levels of learning and satisfaction in the course and also collect demographic data. It is conceivable that demographic and student characteristic variables such as age, gender, and the student's reason for taking courses online, may have an impact on their sense of connectedness and overall learning community. For example, previous research on the relationship between age and social isolation $[17,18]$ indicate higher levels of social isolation with increases in agethus suggesting possible variations in sense of community or connectedness by age. Similar differences have been found in regards to gender [19, 20, 21, 22], with females being less socially isolated than males, suggesting possible variations in scores for sense of community or connectedness by gender. We also believed that respondent's reasons for taking a course, their physical distance from campus, and their employment status may reflect underlying differences in need for, or sense of community as well. Distance from campus and interest in online learning, for example, may be indicators of isolation and could contribute to differences in students' need for a stronger sense of community. Finally, we also felt that the duration of the course could have an impact on participant's sense of community-for example longer courses may provide greater opportunity for creating bonds. These variables were therefore included in the analysis.

\section{PROCEDURES}

Study participants include 2036 students studying in completely online courses in the summer 2004 semester across thirty-two State University of New York colleges in the SUNY Learning Network (SLN). Twenty-one of the institutions were community colleges-reflecting the overall proportion of community colleges to four-year institutions in SLN. There were 470 instructors and 581 courses.

2314 evaluation questionnaires were collected (some students in the sample took more than one course). In all, approximately $20 \%$ or 2181 of the 10,907 students in the summer 2004 group were randomly presented the option of taking the survey when they logged in to their courses. 
The survey was programmed to appear at random two-weeks before the end date of the online course whenever students in the sample logged in. Students in the sample were contacted via email three times by the program administrators with requests to complete the survey when it appeared. The program administrators also enlisted the assistance of the faculty teaching courses during this period asking them to encourage their students to complete the survey. A certain degree of caution needs to be taken in interpreting the results of this survey in light of the fact that only students who completed their courses were included in the sample-i.e. the levels of satisfaction reported here are for students who did not drop out, and may be higher than for non-completers.

\section{RESULTS}

A factor analysis was applied to the data to determine whether the teaching presence and learning community measures cohered into factors reflecting the underlying hypothesized constructs. We would expect to see three factors for the Teaching Presence construct-including instructional design and organization, facilitation of discourse and direct instruction. However, a two-factor solution was highly interpretable-items loaded highly on one factor and low on the other. The analysis reveals that $74.37 \%$ of the variability of the teaching presence construct can be accounted for by this model. The two factors were labeled as "Instructional Design and Organization" and "Directed Facilitation".

There were 2314 respondents to the survey and no missing values were found in the learning community and the teaching presence components. The scores on the learning community and the teaching presence measures were obtained by summing up the items. The means, standard deviations and correlations among the learning community and the teaching presence factors are presented in Table 5 . The mean for total learning community scale was 53.53 out of a possible 80 ; the mean of the connectedness was 24.14 out of a possible score of 40 , the mean of the learning subscale was 29.22 out of a possible 40 . The mean overall score for teaching presence was 52.62 with a maximum score of 68 , the mean score for instructional design and organization was 16.64 with a maximum score of 20, and the mean score for directed facilitation was 35.99 with a maximum score of 48 .

The average scores for the 2314 respondents to the survey were:

\begin{tabular}{|l|c|c|}
\hline & Mean & SD \\
\hline $\begin{array}{l}\text { Total Learning community } \\
\text { (TCC) }\end{array}$ & 53.35 & 12.53 \\
\hline Connectedness & 24.14 & 6.70 \\
\hline Learning & 29.22 & 6.93 \\
\hline Teaching Presence (TP) & 52.62 & 13.75 \\
\hline $\begin{array}{l}\text { Instructional Design and } \\
\text { Organization (IDO) }\end{array}$ & 16.64 & 3.91 \\
\hline Directed Facilitation (DF) & 35.99 & 9.73 \\
\hline
\end{tabular}

Table 1: Average Scores

These average learning community scores, from a large and diverse sample size, can be contextualized by comparing them to results from research with smaller, more purposive samples. Rovai reports results for students' sense of community in traditional, hybrid, and complete online courses that were selected based on the instructors skills in teaching in these different settings. The results for the fully online students $(n=21)$ in Rovai's study were quite a bit higher. 


\begin{tabular}{|c|l|l|}
\hline & Mean & SD \\
\hline Total Learning community (TCC) & 62.29 & 12.53 \\
\hline Connectedness & 29.29 & 8.45 \\
\hline Learning & 33.00 & 6.20 \\
\hline
\end{tabular}

Table 2: Average Scores from Rovai Study

These results appear to be a function of the skill of the instructor in the study reported by Rovai [13], who was selected, as noted by the author, because of his pedagogical abilities. The implication here is that instructors' skills have an impact on online learners' sense of community. In the analysis that follows we analyze which of these skills contributes to online students' sense of learning community.

\section{MULTIPLE REGRESSION ANALYSIS}

A multiple regression analysis was applied to examine the relationship between the learning community measures, the revised teaching presence construct, and demographic information. In the regression analysis, the dependent variable was total learning community as reflected by scores on the Classroom Community Index. The independent variables were instructional design and organization, directed facilitation, and the demographic data. The demographic data included gender, age, employment status, distance from campus, why students took their courses online, students registration status, and the duration of the course. No violations were found in the asssumptions of normality, linearity, and homoscedasticity of residuals. Twenty five outliers were found based on the criteria of beyond \pm 3 standard deviations and removed and thus 2289 cases were used in the present analysis.

The results of the regression model were found to be significant, $\mathrm{F}(21,2288)=183.13, \mathrm{p}<.001$. The multiple correlation coefficient was .79 , indicating that $63 \%$ of total variance of learning community could be accounted for by the revised construct of teaching presence and the demographic characteristic. The constructs instructional design and organization and directed facilitation significantly contributed to the learning community measures. It was found that gender was a significant predictor of learning community as well, although other demographic data were not significant.

All the non-significant predictors were excluded and the regression model was run again. The independent variables used to predict the learning community measure were instructional design and organization, directed facilitation, and gender. Result of analysis of variance indicated that the results of the model were significant, $\mathrm{F}(3,2288)=1259.12, \mathrm{p}<.001$. The correlation coefficients among learning community, the two teaching presence components and gender, the unstandardized beta, standard error and standard beta of the regression model are presented in Table 3.

\begin{tabular}{|c|c|c|c|c|c|c|}
\hline & 1 & 2 & 3 & B & SE B & Beta \\
\hline (Constant) & & & & $17.80^{* * *}$ & .86 & \\
\hline 1. Instructional Design and Organization & 1.00 & & & $.46^{* * *}$ & .07 & .14 \\
\hline 2. Directed Facilitation & $.81^{* * *}$ & 1.00 & & $.81 * * *$ & .03 & .67 \\
\hline 3. Gender & $-.05^{* *}$ & $-.05 *$ & 1.00 & $-.79 *$ & .36 & -.03 \\
\hline
\end{tabular}

Table 3. Correlation Coefficients, Unstandardized Beta, Standard Error, and Standard Beta of the Regression Model of Learning Community 
The multiple correlation coefficient was .79, indicating that $62 \%$ of total variance of learning community could be accounted for by students recognition of effective instructional design and organization, directed facilitation, and by the students' gender. The standardized coefficients were .14, .67, and -.03, which implied that effective "directed facilitation" has a greater contribution in predicting learning community than does instructional design and organization, and female participants tend to have a slightly higher sense of learning community than do their male classmates. A number of conclusions may be drawn from these results - they are discussed below and in more detail in [11].

\section{CONCLUSIONS}

It appears from the analysis presented here that a number of conclusions can be made. The hypothesis that perceived teaching presence is associated with students' sense of learning community was supported. From these results it seems clear that the respondents to the survey were more likely to report a stronger sense of learning community when they also reported that their instructors exhibited stronger "teaching presence” behaviors. In a general sense, when students reported effective instructional design and organization and "directed facilitation" of discourse, as defined by the teaching presence section of the instrument, they were more likely to report higher levels of learning community, as measured by the Classroom Community Scale. A majority of the variance in the scores for this measure of learning community can be explained by the students' sense of their instructors' teaching presence.

It appears that "directed facilitation" on the part of the instructor contributes more to the equation than measures of effective instructional design and organization and gender differences. This study reveals that a strong and active presence on the part of the instructor-one in which she or he actively guides the discourse-is related both to students sense of connectedness and learning. This does not discount the importance of good instructional design and organization. Student who reported more effective instructional design and organization also reported higher levels of learning community-the contribution to the regression equation was simply not as great.

To understand what matters from these results it is useful to recall the components of directed facilitation that contribute to students' sense of connectedness to other course participants and their reports of learning. The components that appear to matter include whether the students feel the instructor is drawing in participants, creating an accepting climate for learning, keeping students on track, and diagnosing student misperceptions. Additionally when students feel their instructors are identifying areas of agreement and disagreement and helping to resolve these by looking for areas of consensus the students report higher levels of connectedness and learning as measured by the Classroom Community Index. Further when students report that the instructor is reinforcing student contributions, injecting their own knowledge, and confirming student understanding, they are also more likely to report a better sense of learning community as measured by the Rovai instrument.

In addition to the directed facilitation outlined above, student perceptions of effective instructional design and organization also appear to matter in regards to a sense of connectedness and learning. The communication of time parameters, due dates, and deadlines contribute to learning community as do clear course goals, clear course topics, and clear instructions on how to effectively and appropriately participate in the course.

In addition to teaching presence behaviors we examined student demographics as they relate to students' sense of community in this online learning environment. Student characteristics that we thought might be of interest included age, gender, employment status, reason for taking the course online, physical distance 
from campus, and previous online learning experience. Brown [23] concluded that experienced students have more time to devote to community building than their newer counterparts and that novice online students require greater interaction with and support from online instructors. We did not find strong evidence to support these hypotheses in the present study though. While there were small differences with regard to gender, the other demographic variables entered in the regression equation did not significantly contribute to the prediction for students' sense of learning community in these online courses. This may come as somewhat of a surprise-it seems reasonable that some of these demographic might serve as proxies for important predictors of ability or desire to participate in a learning community. For example, full-time employment status might indicate that the student is very busy, or already a member of other communities and therefore less likely to recognize and feel part of a learning community in an online course. On the other hand, increased "distance from campus" might reasonably be interpreted as a measure of "academic isolation" and therefore associated with a desire for participation in a learning community. We did not find an indication of such associations here. Students' reports of their instructors teaching presence behaviors far more clearly predicted their sense of online learning community.

Another variable that we thought might provide insight into the development of learning community was course duration-however, we did not find evidence that courses that are of longer duration resulted in a better sense of learning community. It appears from these results that instructor behaviors, as reported by their online students, are a much better predictor of the development of learning community in the online environment. It may be that community develops, not as the result of longer courses, but through the strength of ties developed throughout an entire program—additional research is needed in this area.

The results presented here, with a relatively large sample size, may be considered a benchmark for other institutions seeking to develop high quality online teaching and learning environments. The overall average scores that were reported here may be improved upon by helping faculty to do more of what appears to matter to students, i.e. where learners report higher levels of teaching presence behaviorsactive directed facilitation and effective instructional design and organization. They also report higher levels of learning community. Institutions can use these baseline scores and recommendations to begin to create online learning environments that promote higher levels of both connectedness and learning - two measures that are hallmarks of educational quality, both online and off. If the positive connection between student sense of community and higher retention rates applies to online learning-and there is little reason to doubt that it does - they should also begin to see reduced levels of attrition.

\section{REFERENCES}

1. Garrison, D. R., T. Anderson, and W. Archer. Critical Inquiry in a Text-based Environment: Computer Conferencing in Higher Education. Internet and Higher Education 11(2): 1-14, 2000.

2. Hiltz, S. R. Collaborative Learning in Asynchronous Learning Networks: Building Learning Communities. Paper presented at the WEB98, Orlando, Florida. Online: http://eies.njit.edu/ hiltz /collaborative_learning_in_asynch.htm.

3. Sergiovanni, T. The story of community. In J. Retallick, B. Cocklin, and K. Coombe (Eds.), Learning Communities in Education: Issues, Strategies and Contexts, 9-25. London: Routledge, 1999.

4. Swan, K., and P. Shea. Social Presence and the Development of Virtual Learning Communities. In: Hiltz, S. and Goldman, R. (Eds.), Learning Together Online: Research on Asynchronous Learning Networks, 239-260. Mahwah, NJ: Lawrence Erlbaum, 2005.

5. Palloff, R. N., and K Pratt. Building Learning Communities in Cyberspace. San Francisco: JosseyBass Inc, 1999. 
6. Ludwig-Hardman, S. Case study: Instructional design strategies that contribute to the development of online learning community. Unpublished doctoral dissertation, University of Colorado, Denver, 2003.

7. Bruner, J. The Culture of Education. Cambridge: Harvard University Press, 1996.

8. Barr, R. B. From Teaching to Learning: A New Reality for Community Colleges. Leadership Abstracts. League for Innovation in the Community College 8(3): Mission Viejo, CA, 1995.

9. Barr, R. B. and J. Tagg. From Teaching to Learning-A New Paradigm for Undergraduate Education. Change Magazine 27(6): 12-25, 1995.

10. Schon, D. A. The Reflective Practitioner: How Processionals Think in Action. New York: Basic Books, 1983.

11. Shea, P., A. Pickett, and W. Pelz. A Follow-up Investigation of Teaching Presence in the SUNY Learning Network. The Journal of Asynchronous Learning Networks 7(2): 61-80, 2003.

12. Shea, P., E. Fredericksen, A. Pickett, and W. Pelz. The SUNY Learning Network. In: T. Duffy, and J. Kirkley, (Eds.), Learner Centered Theory and Practice in Distance Education. Mahwah, NJ: Lawrence Erlbaum, 2003.

13. Shea, P. C. S. Li, K. Swan, and A. Pickett. Teaching Presence and Establishment of Community in Online Environments: A Preliminary Study. The Journal of Asynchronous Learning Networks 9(4): 2005.

14. Rovai, A. P. A Preliminary Look at Structural Differences in Sense of Classroom Community Between Higher Education Traditional and ALN Courses. The Journal of Asynchronous Learning Networks 6(1): 41-56, 2002.

15. Rovai, A. P. Development of an Instrument to Measure Classroom Community. Internet and Higher Education 5: 197-211, 2002.

16. Anderson, T., L. Rourke, D. R. Garrison, and W. Archer. Assessing Teaching Presence in a Computer Conferencing Context. Journal of Asynchronous Learning Networks 5(2): 1-17, 2001.

17. Cattan, M., M. White, J. Bond, and A. Learmouth. Preventing Social Isolation and Loneliness Among Older People: A Systematic Review of Health Promotion Interventions. Ageing and Society 25(1): 41-50, 2005.

18. Yeh, S. J., and K. L. Sing. Living Alone, Social Support and Feeling Lonely Among the Elderly. Social Behavior and Personality-An International Journal 32(2): 129-139, 2004.

19. Vandervoort, D. Social Isolation and Gender. Current Psychology 19(3): 229-223, 2000.

20. Shumaker, S. and D. Hill. Gender Differences in Social Support and Physical Health. Health Psychology 10: 102-111, 1991.

21. Bostock, S. and W. Lizhi. Gender in Students' Online Discussions. Innovations in Education and Teaching International. 42(1): 73-86, 2005.

22. Goldstein, J., and P. Sadhana. The Brink of Change: Gender in Technology-Rich Collaborative Learning Environments. Journal of Science Education \& Technology 13(4): 505-523, 2004.

23. Brown, R. The Process of Community Building in Distance Learning Classes. Journal of Asynchronous Learning Networks 5(2): 18-35, 2001.

\section{ABOUT THE AUTHOR}

Peter Shea is a member of the faculty in the Department of Educational Theory and Practice at University at Albany, State University of New York, where he has taught at the graduate level both online and in the classroom. He has a joint appointment in the College of Computing and Information. Previously he was the director of the SUNY Learning Network, the award-winning online education system for the State University of New York. Dr. Shea has also served as manager of the SUNY Teaching, Learning, and Technology Program and as project director for SUNY's participation in the Multimedia Educational Resource for Learning and Online Teaching (MERLOT), an international collaboration for peer review of discipline-specific online learning resources. He is also member of the USNY Technology Policy Council. 
His research focuses on the student and faculty experience in technology-mediated teaching and learning, most recently on the topics of teaching presence and community in asynchronous learning networks. He is the author of many articles and several book chapters on the topic of online learning, co-author of The Successful Distance Learning Student (Thomson-Wadsworth), and a contributor to the new book Learning Together Online: Research on Asynchronous Learning Networks (Lawrence Erlbaum). He is a co-recipient of several awards, including the EDUCAUSE Award for Systemic Progress in Teaching and Learning for the State University of New York, and Sloan Consortium Awards for Excellence in Faculty Development and Asynchronous Learning Networks Programs. He is a member of the American Educational Research Association and the editorial board for the Journal of Asynchronous Learning Networks. His research has appeared in the Journal of Educational Computing Research, The International Review of Research in Open and Distance Learning, and the Journal of Asynchronous Learning Networks. 